\title{
New Approaches for Measuring Electrostatic Potentials and Charge Density Distributions in Working Devices Using Off-Axis and In-Line Electron Holography
}

\author{
Rafal E. Dunin-Borkowski ${ }^{1}$, Vadim Migunov ${ }^{1}$, Andrew London ${ }^{2}$, Michael Farle ${ }^{3}$, Amir H. Tavabi ${ }^{1}$, and \\ Giulio Pozzi ${ }^{4}$ \\ 1 Ernst Ruska Centre for Microscopy and Spectroscopy with Electrons and Peter Gruenberg Institute, \\ Forschungszentrum Juelich, Juelich, Germany \\ 2 Department of Materials, University of Oxford, Parks Road, Oxford, United Kingdom \\ 3 Fakultaet fuer Physik \& Center of Nanointegration, Universitaet Duisburg-Essen, Duisburg, Germany \\ 4 Department of Physics and Astronomy, University of Bologna, Viale B. Pichat 6/2, Bologna, Italy
}

The use of advanced specimen holders with multiple electrical contacts, in combination with off-axis or in-line electron holography, allows electrostatic potentials in nanoscale working devices to be measured quantitatively in the transmission electron microscope (TEM).

Figures 1 and 2 illustrate results obtained by applying off-axis electron holography to measure the potential and field around an electrically-biased Fe atom probe tomography needle. The experiment involved applying a voltage between the needle and a counter-electrode placed a distance of $\sim 400 \mathrm{~nm}$. The phase shift recorded using electron holography was analyzed in two ways: (1) by fitting the phase to a simulation based on two lines of opposite charge density and (2) by using a model-independent approach that involves contour integration of the phase gradient to determine the charge enclosed within the integration contour [1]. In the present study, both approaches required evaluation of the difference between phase images recorded at two applied voltages to subtract the mean inner potential and/ or magnetic contribution to the phase. On the assumption of cylindrical symmetry, the 3D potential and field around the needle were determined from the results, as shown in Fig. 1. Charge density profiles along the needle measured using the two approaches are shown in Fig. 2 and are consistent. However, only the model-independent approach revealed the presence of charge accumulation at the needle apex.

The coherence of the transmission electron microscope that was used to obtain the results shown in Figs. 1 and 2 also allowed other interesting electron-optical phenomena related to in-line electron holography to be observed. When two metallic tips similar to the needle shown in Fig. 1 were placed in front of each other at a separation of $\sim 1 \mu \mathrm{m}$ and a potential difference was applied between them, the combined effect of the fields from the two needles resulted in the formation of highly complex interference patterns in bright-field TEM images acquired out-of-focus, as shown in Fig. 3 for a nominal defocus of $9.5 \mathrm{~mm}$ and a potential difference between the needles of $130 \mathrm{~V}$. The overlapping region has a triangular structure that is similar to the elliptic umbilic diffraction catastrophe.

We are presently developing new techniques to allow off-axis electron holography to be used to obtain results similar to those shown in Figs. 1-3 during ultrafast switching processes in situ in the electron microscope. These developments will be discussed [2].

\section{References:}

[1] M. Beleggia et al, Ultramicroscopy 100 (2010), p. 425.

[2] We are grateful to M. Beleggia, T. F. Kelly and D. J. Larson for valuable discussions. 


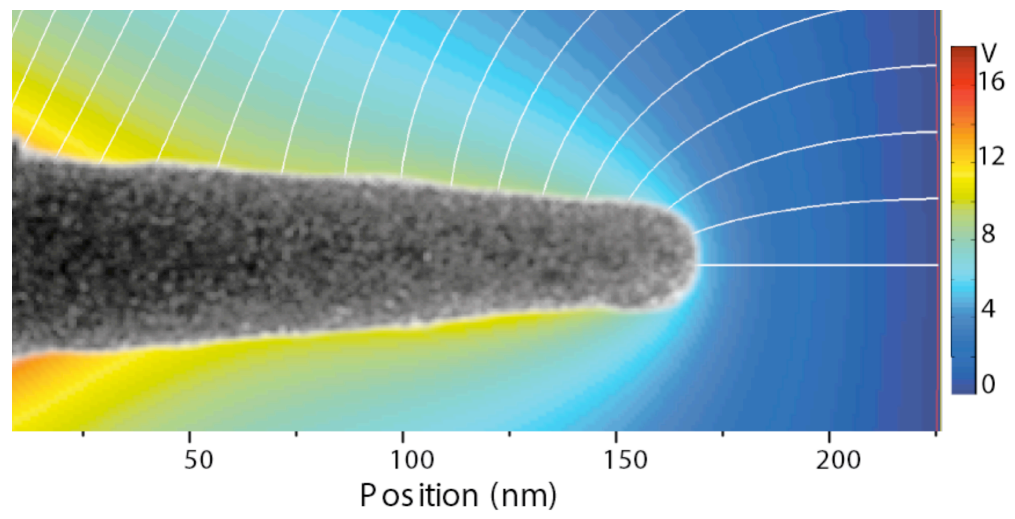

Figure 1. Central slice of the 3D electrical potential (colors) and electric field (white lines) between an $\mathrm{Fe}$ atom probe tomography needle and a $\mathrm{Au}$ counter-electrode, determined from the charge density distribution in the needle measured from the difference between phase images recorded using off-axis electron holography at applied bias voltages between the needle and the counter-electrode of 0 and $5 \mathrm{~V}$.

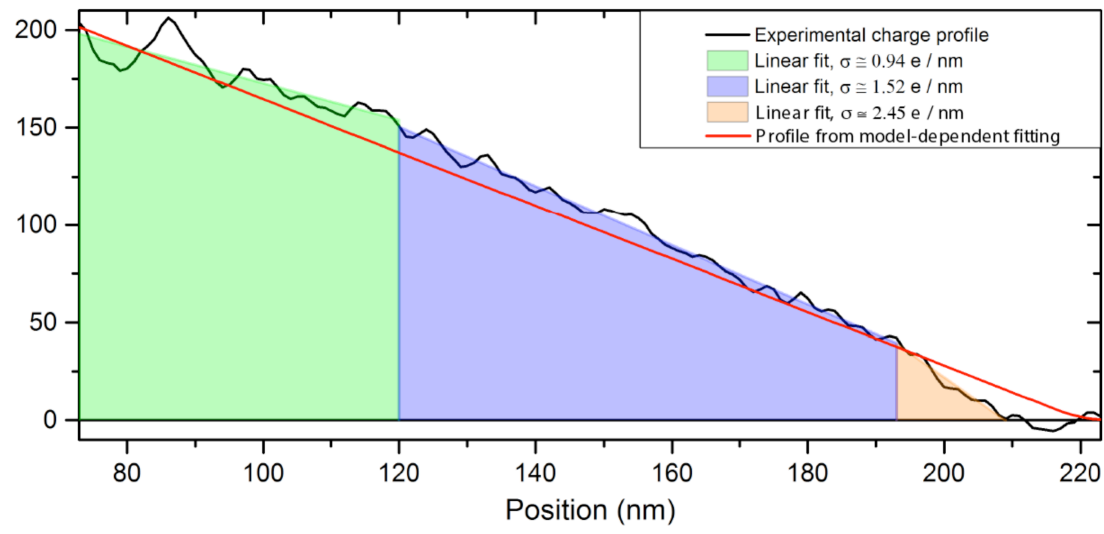

Figure 2. Cumulative charge profiles along the needle shown in Fig. 1, measured by off-axis electron holography in units of electrons using model-independent (black) and model-dependent (red) methods.
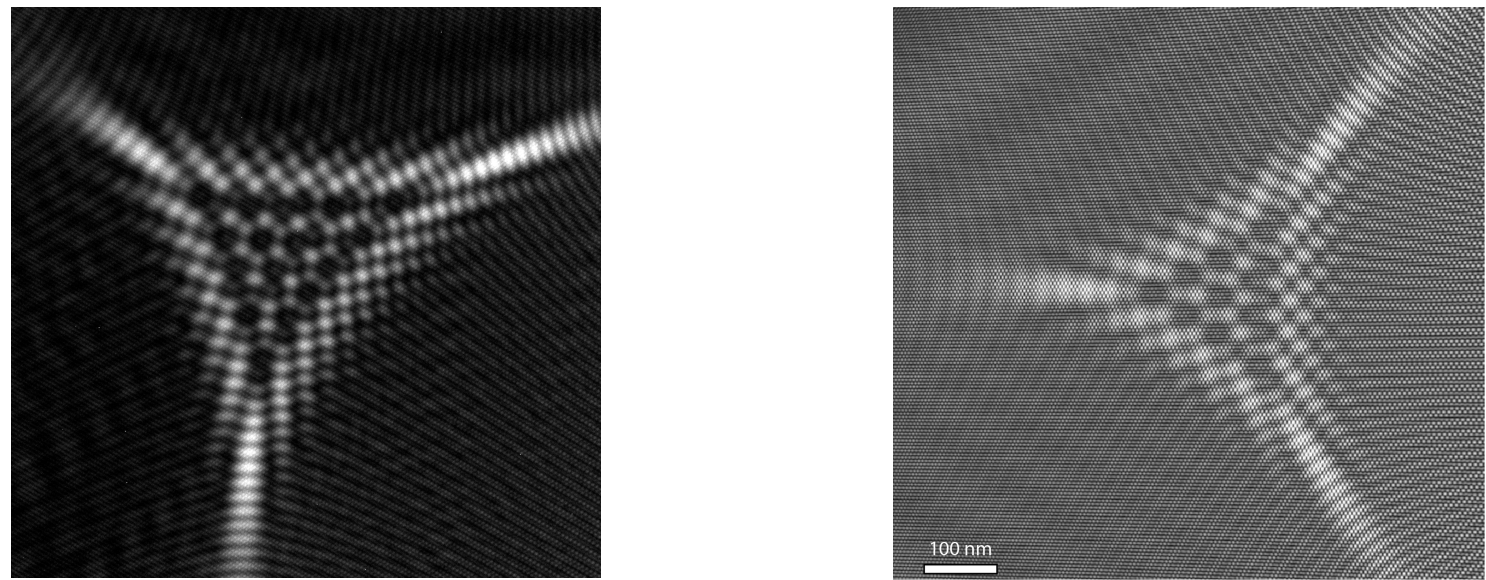

Figure 3. (a) Bright-field TEM image recorded at a defocus of $9.5 \mathrm{~mm}$ from two metallic needles similar to that shown in Fig. 1, with a potential difference of $130 \mathrm{~V}$ between them, showing hexagonallike spots in a caustic. (b) Simulation performed for the experimental conditions used to acquire (a). 